\title{
EVOLUTION OF FAST MAGNETOACOUSTIC PULSES IN RANDOMLY STRUCTURED CORONAL PLASMAS
}

\author{
D. Yuan ${ }^{1,2,3}$, D. J. Pascoe ${ }^{4}$, V. M. NaKariakov ${ }^{4,5,6}$, B. Li $^{1}$, and R. KePpens ${ }^{2}$ \\ ${ }^{1}$ Shandong Provincial Key Laboratory of Optical Astronomy and Solar-Terrestrial Environment, Institute of Space Sciences, \\ Shandong University, Weihai 264209, China; Ding.Yuan@wis.kuleuven.be, bbl@ sdu.edu.cn \\ ${ }^{2}$ Centre for mathematical Plasma Astrophysics, Department of Mathematics, KU Leuven, Celestijnenlaan 200B bus 2400, B-3001 Leuven, Belgium \\ ${ }^{3}$ Key Laboratory of Solar Activity, National Astronomical Observatories, Chinese Academy of Sciences, Beijing 100012, China \\ ${ }^{4}$ Centre for Fusion, Space and Astrophysics, Department of Physics, University of Warwick, Coventry CV4 7AL, UK \\ ${ }^{5}$ School of Space Research, Kyung Hee University, Yongin, 446-701 Gyeonggi, Korea \\ ${ }^{6}$ Central Astronomical Observatory of the Russian Academy of Sciences at Pulkovo, 196140 St Petersburg, Russia \\ Received 2014 October 11; accepted 2014 December 8; published 2015 January 30
}

\begin{abstract}
We investigate the evolution of fast magnetoacoustic pulses in randomly structured plasmas, in the context of large-scale propagating waves in the solar atmosphere. We perform one-dimensional numerical simulations of fast wave pulses propagating perpendicular to a constant magnetic field in a low- $\beta$ plasma with a random density profile across the field. Both linear and nonlinear regimes are considered. We study how the evolution of the pulse amplitude and width depends on their initial values and the parameters of the random structuring. Acting as a dispersive medium, a randomly structured plasma causes amplitude attenuation and width broadening of the fast wave pulses. After the passage of the main pulse, secondary propagating and standing fast waves appear. Width evolution of both linear and nonlinear pulses can be well approximated by linear functions; however, narrow pulses may have zero or negative broadening. This arises because narrow pulses are prone to splitting, while broad pulses usually deviate less from their initial Gaussian shape and form ripple structures on top of the main pulse. Linear pulses decay at an almost constant rate, while nonlinear pulses decay exponentially. A pulse interacts most efficiently with a random medium with a correlation length of about half of the initial pulse width. This detailed model of fast wave pulses propagating in highly structured media substantiates the interpretation of EIT waves as fast magnetoacoustic waves. Evolution of a fast pulse provides us with a novel method to diagnose the sub-resolution filamentation of the solar atmosphere.
\end{abstract}

Key words: magnetohydrodynamics (MHD) - methods: numerical - Sun: atmosphere - Sun: corona Sun: oscillations - waves

\section{INTRODUCTION}

Magnetohydrodynamic (MHD) waves play an important role in the formation and dynamics of the solar atmosphere. They may contribute significantly to coronal heating (see reviews by Klimchuk 2006; Parnell \& De Moortel 2012), and solar wind acceleration and heating (see, e.g., Suzuki \& Inutsuka 2006; Li \& Li 2007, 2008; Ofman 2010). In a uniform magnetized plasma, there are four MHD eigenmodes, disregarding the directions of propagation: Alfvén, fast and slow magnetoacoustic modes, and entropy mode, see Goedbloed \& Poedts (2004). Only the fast magnetoacoustic mode can propagate in a direction perpendicular to the magnetic field, and therefore can experience a wealth of plasma structuring. Plasma structuring significantly modifies MHD wave propagation, causing the effects of wave-guiding, dispersion, linear coupling, resonant absorption, and phase mixing (see, e.g., Edwin \& Roberts 1983; Van Doorsselaere et al. 2008b; Sakurai et al. 1991; Heyvaerts \& Priest 1983; Goedbloed \& Poedts 2004; Goedbloed et al. 2010). Various MHD eigenmodes of regularly structured plasma non-uniformities are well understood theoretically, and are confidently detected at different heights of the solar atmosphere (see reviews by Nakariakov \& Verwichte 2005; Ofman 2005; De Moortel \& Nakariakov 2012; Pascoe 2014). MHD wave theory in regular plasma nonuniformities created a solid basis for the rapid development of the wave-based plasma diagnostics technique-MHD coronal seismology (see Stepanov et al. 2012 for a recent review). MHD seismology has been successfully applied to measuring the magnetic field (Nakariakov \& Ofman 2001), or quantifying transverse loop structuring (Aschwanden et al. 2003),
Alfvén transit times (Arregui et al. 2007), polytropic index (Van Doorsselaere et al. 2011), thermal conduction coefficient (Ofman \& Wang 2002), the magnetic topology of sunspots (Yuan et al. 2014b, 2014a), and the magnetic structure of largescale streamers (Chen et al. 2010, 2011).

On the other hand, MHD waves in a randomly structured plasma are less commonly studied. Before the commissioning of high-resolution EUV observations of the solar corona, the main interest in the effect of random structuring on MHD waves was connected with the interstellar medium and sunspot applications. In particular, Valley $(1971,1974)$ analytically demonstrated that linear MHD waves experience attenuation in a medium with a stochastic magnetic field. Li \& Zweibel (1987) analytically considered the propagation of an Alfvén wave package in a medium with time-dependent short-wavelength random density irregularities and found that the Alfvén waves transferred power into compressive modes that dissipate and heat the plasma. Lazzaro et al. (2000) further developed the analytical theory of linear MHD waves in randomly structured media, and applied it to a specific case of a "two-phase plasma" that consisted of randomly spaced regions with two alternative Alfvén speeds. The scattering of acoustic waves that interact with a bundle of field-aligned fibrils was comprehensively studied by Bogdan \& Zweibel (1985, 1987). Keppens et al. (1994) studied the multiple scattering of acoustic waves in fibril spaghetti sunspots and showed that impinging $p$-modes suffer enhanced damping due to resonant absorption in successive scatters (also see Labonte \& Ryutova 1993; Ryutova \& Priest 1993b, 1993a). Fast magnetohydrodynamic modes of multifibril bundles, representing coronal loops, were considered by 
Díaz et al. (2005). Gruszecki et al. (2006) modeled the effect of random structuring on the period, decay time and amplitude of standing kink modes of a plasma cylinder. Sausage oscillations of a randomly multi-shelled coronal loop were numerically modeled by Pascoe et al. (2007) who found that the period of oscillation was insensitive to very fine structuring. Random structuring was considered in the context of the damping rate (Pascoe et al. 2011) and effect of line-of-sight integration (De Moortel \& Pascoe 2012) for propagating kink waves undergoing mode coupling (Pascoe et al. 2010).

An analytical model based on the eikonal formalism was developed for describing linear shear Alfvén waves in a medium with a randomly tangled magnetic field (Similon \& Sudan 1989). It was found that random structuring of the field affects the wave dissipation significantly by phase mixing. Nonlinear selfinteraction of broadband shear Alfvén waves, which effectively constitute a randomly structured medium for the propagation of individual spectral components, was modeled in terms of reduced MHD (Kleva \& Drake 1992). The efficient dissipationless damping of the waves was established. A similar conclusion was reached by Malara et al. (2003) who analytically described the propagation of short-wavelength small-amplitude Alfvén waves in a randomly structured cold compressive plasma in terms of the WKB approximation. Enhanced damping of Alfvén waves in a plasma with a balanced MHD turbulence was also evident in the incompressible ideal MHD modeling performed by Beresnyak \& Lazarian (2008). Recently, Tsiklauri (2014) modeled numerically the dynamics of linearly polarized Alfvén waves in a randomly tangled magnetic field, and found that the wave evolution is strongly affected by the random structuring.

The discovery of global coronal waves, also known as EIT, EUV or coronal Moreton waves, or coronal bright fronts (Thompson et al. 1999), stimulated further interests in understanding the interaction of MHD waves with a randomly structured coronal plasma. These waves are usually triggered by flares or coronal mass ejections and propagate for several hundred megameters as EUV, soft X-ray, and radio emission intensity disturbances at speeds of several hundred kilometers per second (see Warmuth 2011; Gallagher \& Long 2011; Patsourakos \& Vourlidas 2012 for recent reviews). EIT waves are usually interpreted as fast magnetoacoustic waves (see Wang 2000; Wu et al. 2001, 2005; Ofman \& Thompson 2002 for a discussion), while alternative interpretations have not been ruled out (see, e.g., Delannée 2000; Chen et al. 2005; Patsourakos $\&$ Vourlidas 2012). However, the deceleration, profile broadening, amplitude attenuation (Warmuth et al. 2004a, 2004b; Long et al. 2011b), and nonlinear steepening (Veronig et al. 2010; Shen \& Liu 2012) of EIT waves appear to be consistent with a fast wave nature. Therefore, one could model the interaction of EIT waves with bundles of solar coronal loops as fast magnetoacoustic wave propagation through a randomly structured medium. Wave modification by perpendicular structuring of coronal plasma is demonstrated by the propagation of kink waves (Van Doorsselaere et al. 2008a). In the context of EIT waves, it is important to consider wave pulses rather than harmonic waves. The nonlinear effects connected with the finite amplitude of the waves should also be taken into account. Nakariakov et al. (2005) studied the formation of a dispersive oscillatory wake in a fast magnetoacoustic pulse propagating through a randomly structured medium. Murawski et al. (2001) investigated a possible decrease in the phase speed of the EIT wave in terms of fast MHD wave propagation in a cold plasma with random structuring.
In this study, we use a one-dimensional (1D) ideal MHD model to simulate the propagation of fast magnetoacoustic pulses perpendicular to the magnetic field in a randomly structured plasma. The structuring is applied across the field direction, and the parametric values are set in accordance with coronal conditions. The purpose of our study is to understand amplitude attenuation and broadening of the pulses caused by the random structuring. Our model differs from the study by Murawski et al. (2001) in a number of ways. (1) We use a random structuring of a uniform spectrum in contrast to a Gaussian spectrum. (2) Murawski et al. (2001) constructed a random profile by evaluating the Alfvén speed directly. This means extra mass is loaded to the system by the structuring in comparison to a uniform plasma. This step naturally leads to the retardation of the main pulse. (3) Murawski et al. (2001) consider only the linear wave regime in an incompressible (zero$\beta$ ) plasma, while we study both linear and nonlinear regimes in typical compressible coronal plasmas. This paper is organized as follows; the numerical model is described in Section 2. The simulation results are presented in Section 3, followed by our conclusions (Section 4).

\section{NUMERICAL MODEL}

We use the MPI-AMRVAC code (Keppens et al. 2012; Porth et al. 2014) to solve a set of normalized ideal MHD equations in conservative form using a finite volume method,

$$
\frac{\partial \mathbf{U}}{\partial t}+\nabla \cdot \mathbf{F}(\mathbf{U})=0
$$

where

$$
\mathbf{U}=\left[\begin{array}{c}
\rho \\
\rho \mathbf{v} \\
e \\
\mathbf{B}
\end{array}\right] \text {, and } \mathbf{F}=\left[\begin{array}{c}
\rho \mathbf{v} \\
\mathbf{v} \rho \mathbf{v}+\mathbf{I}\left(p+\mathbf{B}^{2} / 2\right)-\mathbf{B B} \\
\mathbf{v}\left(e+p+\mathbf{B}^{2} / 2\right)-\mathbf{v} \cdot \mathbf{B B} \\
\mathbf{v B}-\mathbf{B} \mathbf{v}
\end{array}\right]
$$

are the conservative variables and flux functions, respectively. Furthermore, $\rho$ is the mass density, $\mathbf{v}$ is the plasma velocity, $\mathbf{B}$ is the magnetic field, $e=p /(\gamma-1)+\rho \mathbf{v}^{2} / 2+\mathbf{B}^{2} / 2$ is total energy density, $p$ is the gas pressure, $\gamma$ is the adiabatic index, and $\mathbf{I}$ is a $3 \times 3$ unit matrix. Adiabaticity is assumed, namely, the specific entropy is conserved in the Lagrangian frame $\left(\mathrm{d}\left(p \rho^{-\gamma}\right) / \mathrm{d} t=0\right)$.

The MHD equations (Equation (1)) are solved in Cartesian coordinates $(x, y, z)$ by assuming that the physical quantities do not vary along the $x$ and $z$ directions $(\partial / \partial x=\partial / \partial z=0)$. Therefore, the MPI-AMRVAC code is configured to a $1.5 \mathrm{D}$ MHD setup. We use an idealized 1D plasma model with a constant magnetic field and a random density profile perpendicular to the magnetic vector. This kind of approximation was successfully applied using the LARE2D code (Arber et al. 2001) to model 1D MHD processes in the solar atmosphere (e.g., Tsiklauri et al. 2004; Botha et al. 2011). In the following text, we consistently adopt dimensionless quantities. However, one could restore the dimensionalities by choosing any set of three independent variables, e.g., $B_{0}=10 \mathrm{G}, L_{0}=1000 \mathrm{~km}$, $\rho_{0}=7.978 \times 10^{-13} \mathrm{~kg} \mathrm{~m}^{-3}$, which are typical values for coronal plasmas. The Alfvén speed $V_{A}=B_{0} / \sqrt{\rho_{0} \mu_{0}}=1000 \mathrm{~km} \mathrm{~s}^{-1}$ (where $\mu_{0}=4 \pi \times 10^{-7} \mathrm{~N} \mathrm{~A}^{-2}$ is the magnetic permeability of free space) and Alfvén transit time $\tau_{A}=L_{0} / V_{A}=1 \mathrm{~s}$ serve as the units for the velocity and time, respectively.

The magnetic field was chosen to be constant and in the $x$ direction $\mathbf{B}=(1,0)$. The density profile consists of a set of 

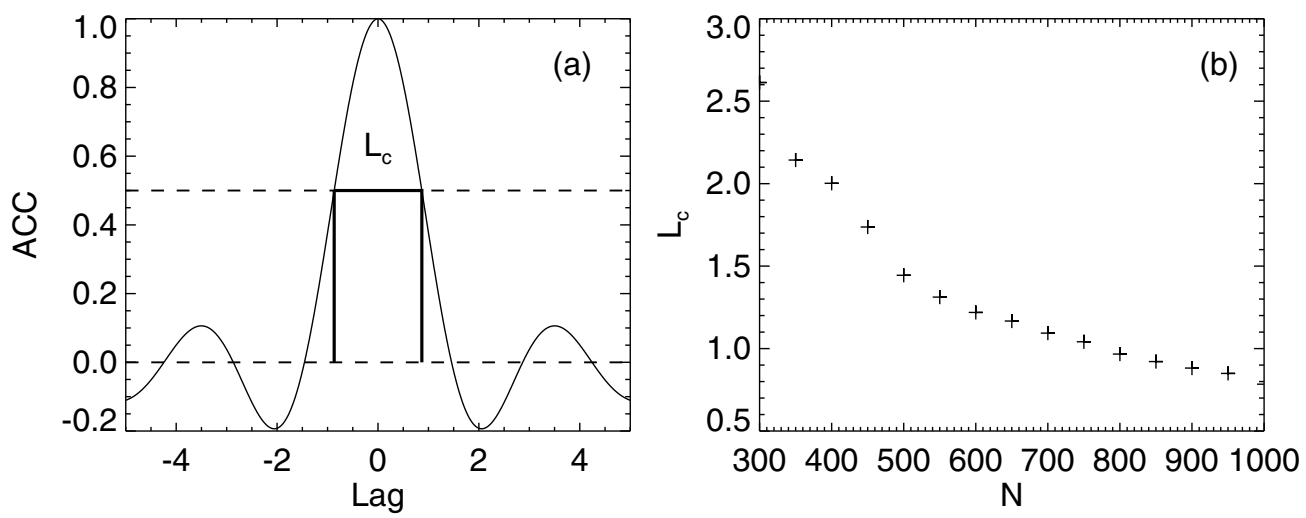

Figure 1. (a) The correlation length $L_{c}$ of a randomly structured plasma defined as the FWHM of the autocorrelation coefficient (ACC) of the profile. The dashed lines present the zero and half-maximum levels of ACC. (b) Dependence of the correlation length $L_{c}$ on the number of harmonics $N$. The correlation length $L_{c}$ decreases as higher harmonics are added to the density profile.
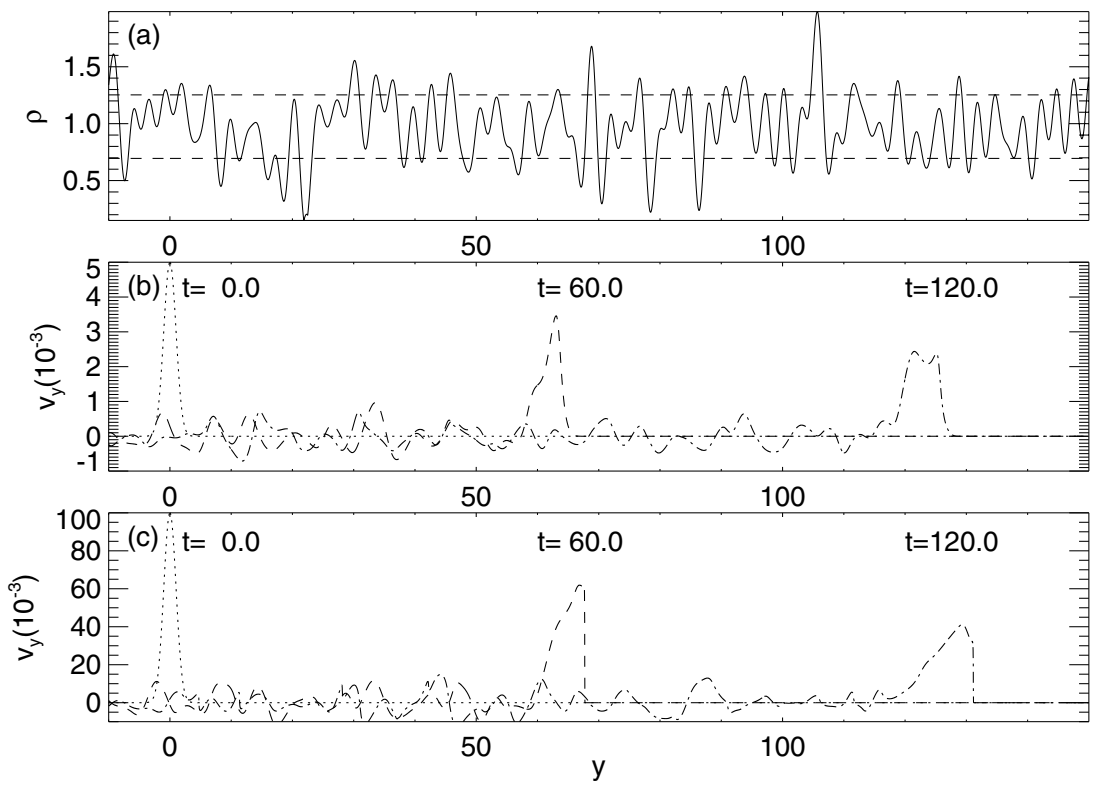

Figure 2. (a) Density profile for a randomly structured plasma with a density contrast $\delta_{\rho}=0.28$ and a correlation length $L_{c}=1.46$. The dashed lines mark the density levels at $1 \pm \delta_{\rho}$. Small- ((b), $\left.A_{0}=0.005\right)$ and large-amplitude $\left((\mathrm{c}), A_{0}=0.1\right)$ fast magnetoacoustic pulses of width $w_{0}=2.35$ were launched and demonstrate linear and nonlinear wave effects. The snapshots of the pulse evolution are overplotted at $t=0$ (dotted lines), $t=60$ (dashed lines), and $t=120$ (dot-dashed lines).

sinusoidal harmonic perturbations in the $y$ direction added to a constant background:

$$
\rho(y)=1+\frac{\Delta}{N} \sum_{i=1}^{N} R_{i} \sin \left(\alpha k_{i} y+\phi_{i}\right),
$$

where $\Delta$ is a scaling parameter to control the density contrast $\delta_{\rho}=\left[\int_{0}^{L_{y}}(\rho(y)-\bar{\rho})^{2} \mathrm{~d} y / L_{y}\right]^{0.5}$, in which $\bar{\rho}=$ $\int_{0}^{L_{y}} \rho(y) \mathrm{d} y / L_{y} \simeq 1$ is the mean density, and $L_{y}$ is the size of the numerical domain in the $y$ direction. The parameter $k_{i}=i \pi / L_{y}$ is the wavenumber of the $i$ th harmonic component. The values $R_{i}$ and $\phi_{i}$ are the random amplitude and phase of the $i$ th harmonic component, given by a uniform pseudo-random number generator within the ranges $[0,1]$ and $[-\pi, \pi]$, respectively. The number of harmonics $N$ determines the correlation length $L_{c}$ of the density profile. The factor $\alpha$ controls how fast $L_{c}$ drops off with increasing $N$, and we take $\alpha=1 / 4$ in our study. Figure 1 shows the definition of the correlation length $L_{c}$ of the random structuring as the FWHM of the au- tocorrelation function of the density profile. This value can be monotonically controlled by adding more harmonics (i.e., increasing $N$ ) to the density profile (Equation (2)). Figure 2(a) illustrates a typical density profile with $\delta_{\rho}=0.28$ and $L_{c}=1.26(N=600)$, which implies that about $67 \%$ of the density humps is distributed within $28 \%$ of the mean value in the density profile and that they are separated by a distance of $L_{c}$ on average. This scenario could also be viewed as bundles of coronal loops with average widths of $L_{c}$, filled with plasmas of random density that fluctuate around the mean value $\bar{\rho}$ with a standard deviation of $\delta_{\rho}$.

The computational domain size $L_{y}$ is set to be 160 . We apply open boundary conditions at both sides of the $y$ dimension, so that energy is free to propagate out of the simulation domain. In practice, this is implemented by zeroth-order extrapolation of the conservative variables in the ghost cells in combination with the exploited approximate Riemann solver; this also ensures no artificial wave reflection at the boundaries. In the initial equilibrium the plasma $\beta=0.01$ is enforced over the simulation domain. In the initial state total pressure balance 
$P_{\text {total }}(y)=\mathbf{B}^{2} / 2+\rho(y) T(y)=(1+\beta) \mathbf{B}^{2} / 2$ is ensured by adjusting the temperature profile $T(y)$ accordingly, meaning that the temperature profile is also randomly structured.

A fast magnetoacoustic pulse is launched by augmenting the equilibrium values with a perturbation to the initial conditions as follows,

$$
\begin{aligned}
& v_{y}^{\prime}(y)=A_{0} \exp \left[-4 \ln 2 \frac{\left(y-y_{0}\right)^{2}}{w_{0}^{2}}\right], \\
& B_{x}^{\prime}(y)=A_{0} \exp \left[-4 \ln 2 \frac{\left(y-y_{0}\right)^{2}}{w_{0}^{2}}\right], \\
& \rho^{\prime}(y)=A_{0} \exp \left[-4 \ln 2 \frac{\left(y-y_{0}\right)^{2}}{w_{0}^{2}}\right],
\end{aligned}
$$

where $v_{y}^{\prime}, B_{x}^{\prime}$, and $\rho^{\prime}$ are the perturbations of the $y$ component of the velocity, the $x$ component of the $\mathbf{B}$ field and plasma density, respectively. Furthermore, $y_{0}=0$ is the starting position of the pulse, and $A_{0}$ and $w_{0}$ are the initial amplitude and width (FWHM) of the pulse, respectively. This specific form of perturbation ensures that the fast pulse will propagate in one direction.

Equation (1) is solved with the HLL approximate Riemann solver (Harten et al. 1983) and a three-step Runge-Kutta method in time discretization (for details, see Keppens \& Porth 2014; Porth et al. 2014 and references therein). HLL is an upwind Godunov-type scheme that uses a constructed flux function with two of the fastest wave speeds and switches the flux accordingly to the orientation of the Riemann fan in the $y-t$ space. We performed a feasibility study to see how the use of a different limiter exploited in the cell center-to-edge variable reconstruction behaves in the wave problem by launching a linear wave pulse with $A_{0}=0.005$ and $w_{0}=0.94$ in a uniform magnetized plasma $(\Delta=0$ in Equation (2)). We switched between possible limiter options in MPI-AMRVAC and assessed their quality by examining how much the wave amplitude is numerically damped by the combined effects of the approximate Riemann solver, time advancing, and reconstruction limiter. These simulations were run with 9600 fixed grid cells $(\Delta y=0.0167)$. After traveling a distance of 150 , the fast wave pulse was damped by $25.0 \%$ (MINMOD), 9.6\% (WOODWARD), $12.0 \%$ (ALBADA), $10.8 \%$ (MCBETA), $6.1 \%$ (KOREN), $5.8 \%$ (PPM), and $-5.1 \%$ $\left(\right.$ cada3 $^{7}$ ) (see details in, e.g., Tóth \& Odstrčil 1996; Kuzmin 2006; Keppens \& Porth 2014 and references therein). We exclude the limiters that either cause significant numerical damping (MINMOD, WOODWARD, ALBADA, and MCBETA) or result in numerical artefacts (CADA3). Furthermore, because PPM requires four ghost cells rather than two and is therefore computationally more expensive, we opt for the KOREN flux limiter (Koren 1993) and use 12,000 fixed grid cells. The wave is then damped by about $5 \%$ for $w_{0}=0.94$, about $1 \%$ for $w_{0}=1.18-1.41$, and less than $0.1 \%$ for $w_{0}>1.88$ for pulses propagating in a uniform medium. Note that these numerical errors are overestimated, if we must take into account that the initial pulse setup is not an exact eigenmode solution of the system and that we quantified errors by assuming unchanged pulse shape. In the uniform medium, the pulse does not experience broadening or develop the oscillatory wake.

\footnotetext{
7 Note: a negative damping means that CADA3 is too strong of a limiter and results in the amplification of the pulse.
}

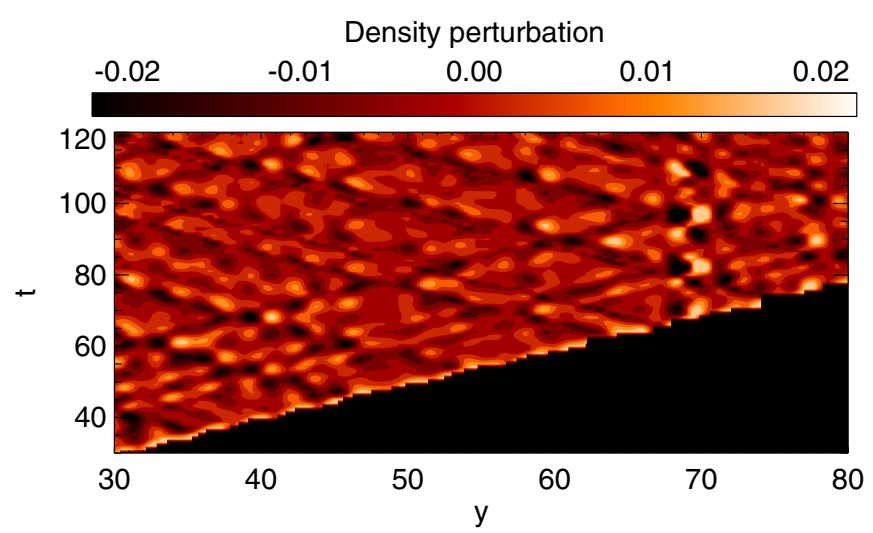

Figure 3. Baseline-difference time-distance plot for the density perturbations $\rho(y, t)-\rho(y, 0)$ showing the secondary pulses trapped by a randomly structured plasma after the passage of a small-amplitude fast magnetoacoustic pulse. This is the same run as in Figure 2(b). The blank bottom right triangular region represents the passage of the main pulse and the associated density perturbation is masked out.

\section{RESULTS}

Figure 2 presents snapshots of the evolution of both a linear and a nonlinear fast magnetoacoustic pulse of the same initial width. Consistent with previous findings discussed in Section 1, the random structuring causes both attenuation and dispersion. Nonlinear pulses experience steepening, but the efficiency is reduced by effective attenuation and dispersion caused by the random structuring.

Figure 3 shows a typical baseline-difference time-distance plot (see Yuan \& Nakariakov 2012 for technical details) for a fast pulse propagating through a randomly structured medium. The main pulse was covered by a dynamic mask (black region), and hence small-amplitude density perturbations (colored region) were revealed to illustrate the plasma evolution in the aftermath of the main pulse. Figure 3 reveals two kinds of perturbations. The diagonal ridges represent secondary pulses propagating approximately at the average Alfvén speed in both directions. The average Alfvén speed also corresponds to the slope of the line indicating the main pulse, which travels at a speed of about unity in our simulations. These pulses appear due to reflections from the non-uniformities. Standing features (see, e.g., $y \simeq 69$ ) that oscillate in time correspond to the eigenmodes of the non-uniformities excited by the main and secondary pulses. The energy that excites secondary propagating and standing features was extracted from the main pulse causing its gradual decay.

To quantify the effect of the structuring, we measured the amplitude $A(y)$ and width $w(y)$ of the peak in the velocity perturbation profile for each snapshot. Figure 4 shows the evolution of pulse amplitude and width for both a linear (asterisk) and a nonlinear (diamond) wave. The widths of both linear and nonlinear pulses increase quasi-linearly with propagation distance, so we obtain a characteristic width amplification coefficient $K_{s}$ by fitting the data with $w / w_{0}=1+K_{s} y .{ }^{8}$ The amplitude of a linear pulse is damped linearly by the random structuring, while the nonlinear pulse decays exponentially; thus, we fit them with $A / A_{0}=1-K_{a} y$ and $A / A_{0}=\exp \left(-K_{a}^{*} y\right)$, respectively. Note that $\exp \left(-K_{a}^{*} y\right) \simeq 1-K_{a}^{*} y$ for $K_{a}^{*} \ll 1$. In the following text and figures, $K_{a}$ denotes both linear and exponential damping coefficients, where we drop the superscript for the latter.

\footnotetext{
8 We used MPFIT (Markwardt 2009) package to perform all of the fittings, the source code is available at http://purl.com/net/mpfit.
} 

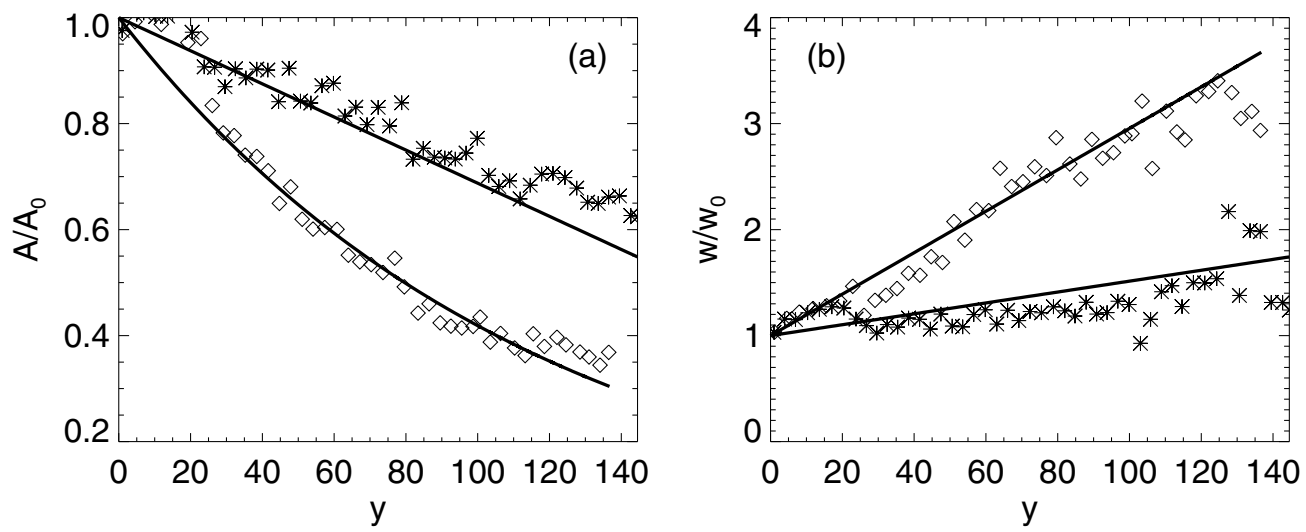

Figure 4. Linear $\left(A_{0}=0.005\right.$, asterisk $)$ and nonlinear $\left(A_{0}=0.1\right.$, diamond $)$ fast magnetoacoustic pulses launched with initial widths of $w_{0}=1.9$ in a randomly structured plasma with a density contrast $\delta_{\rho}=0.24$ and a correlation length $L_{c}=1.3$. Panels (a) and (b) plot the normalized amplitude and width evolution (symbols), respectively. An exponential fit is applied to the amplitude evolution of the nonlinear pulse, and other profiles were fitted with a linear function (solid lines).
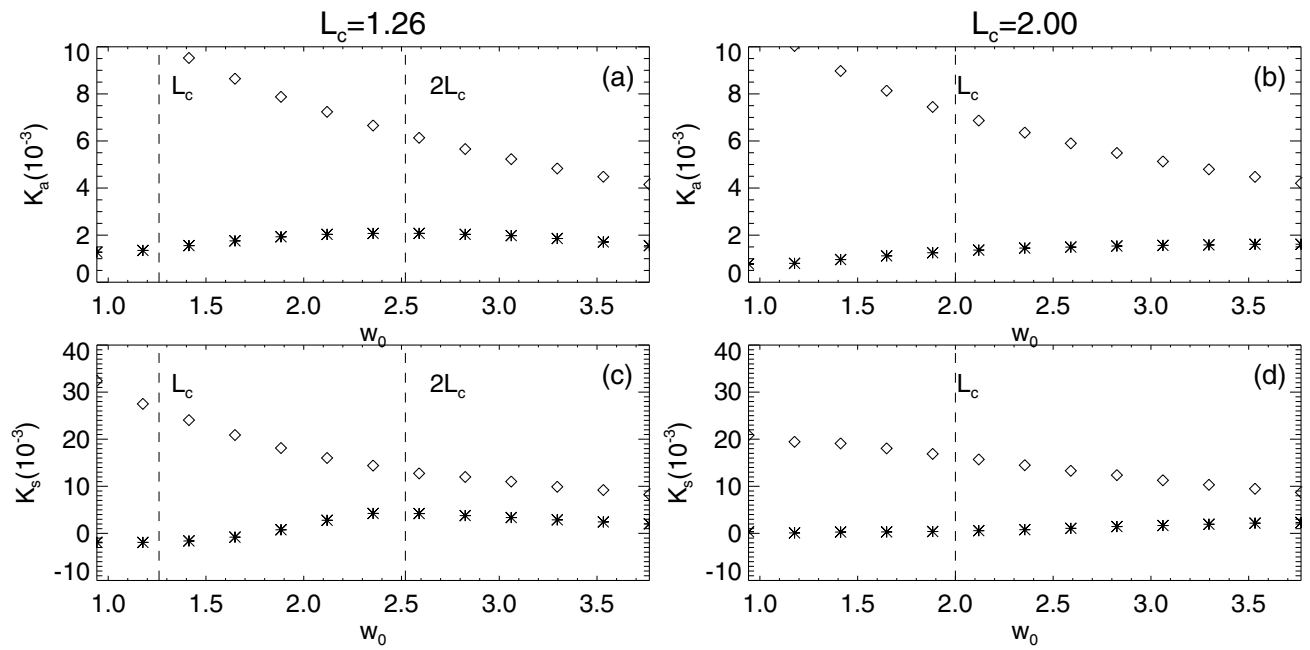

Figure 5. $K_{a}$ and $K_{s}$ as functions of the initial pulse width $w_{0}$ for the linear (asterisks) and nonlinear (diamonds) waves in a randomly structured plasma with density contrasts of $\delta_{\rho}=0.18$ and correlation lengths of $L_{c}=1.26(\mathrm{a}, \mathrm{c})$ or $L_{c}=2.0(\mathrm{~b}, \mathrm{~d})$.

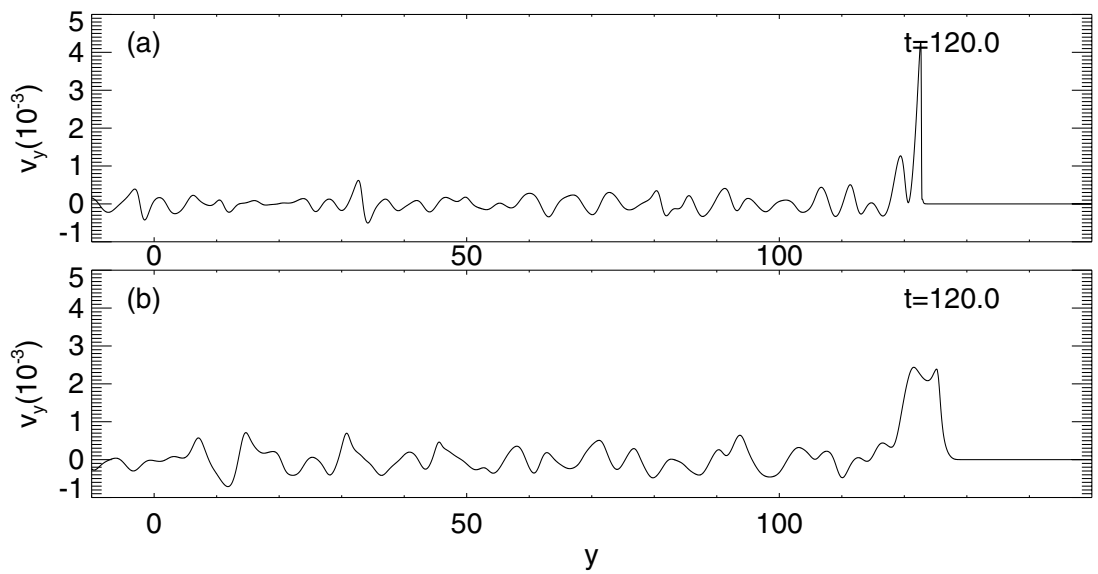

Figure 6. (a) A narrow pulse $\left(w_{0}=1.17\right)$ splits into a main pulse and a co-evolving subpulse. (b) A broad pulse $\left(w_{0}=2.35\right)$ exhibits sub-structuring on top of its main pulse.

In the case of Figure 4, we obtain $K_{a}=0.0031$ and 0.0087 for the linear and nonlinear amplitude evolutions, respectively, while the width profiles $K_{s}=0.0051$ and 0.0196 were found. The coefficients $K_{s}$ and $K_{a}$ depend on the initial parameters of the pulse (width $w_{0}$ ), and on the parameters of random structuring (density contrast $\delta_{\rho}$ and correlation length $L_{c}$ ). To investigate this behavior, we performed a set of parametric studies.

\subsection{Effect of Initial Pulse Width}

We first consider the effect of the initial pulse width $w_{0}$. Figure 5 shows that nonlinear pulses $\left(A_{0}=0.1\right)$ are attenuated stronger than linear ones $\left(A_{0}=0.005\right)$ and their widths also expand faster, i.e., the $K_{a}$ and $K_{s}$ values are larger. For a linear pulse in a random plasma with $L_{c}=1.26$, the maximum 

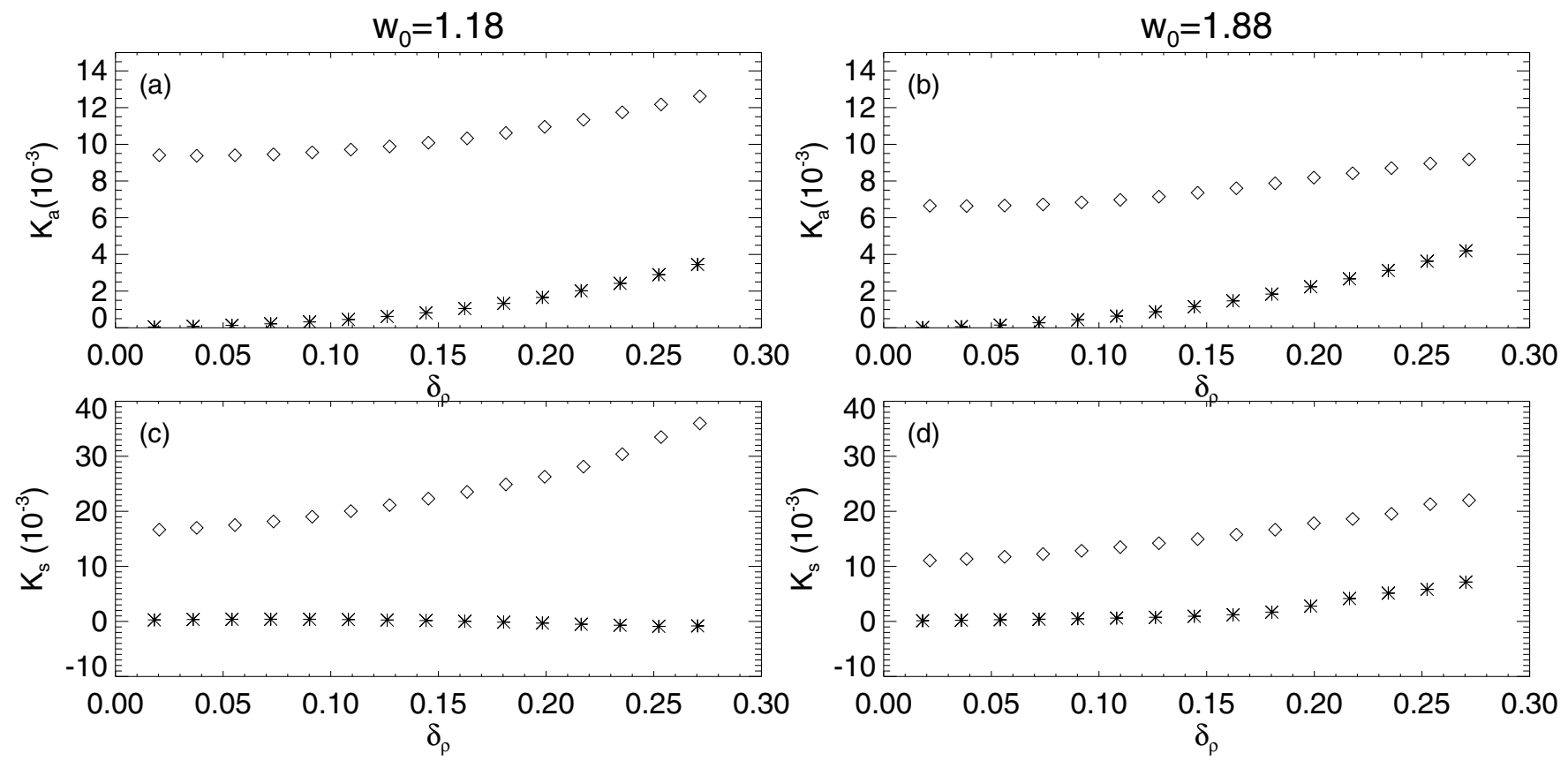

Figure 7. $K_{a}$ and $K_{s}$ as functions of the density contrast $\delta_{\rho}$ of the random structuring with $L_{c}=1.26$ for the linear (asterisks) and nonlinear (diamonds) waves. Two sets of pulses were launched with $w_{0}=1.18(\mathrm{a}, \mathrm{c})$ or $w_{0}=1.88(\mathrm{~b}, \mathrm{~d})$.
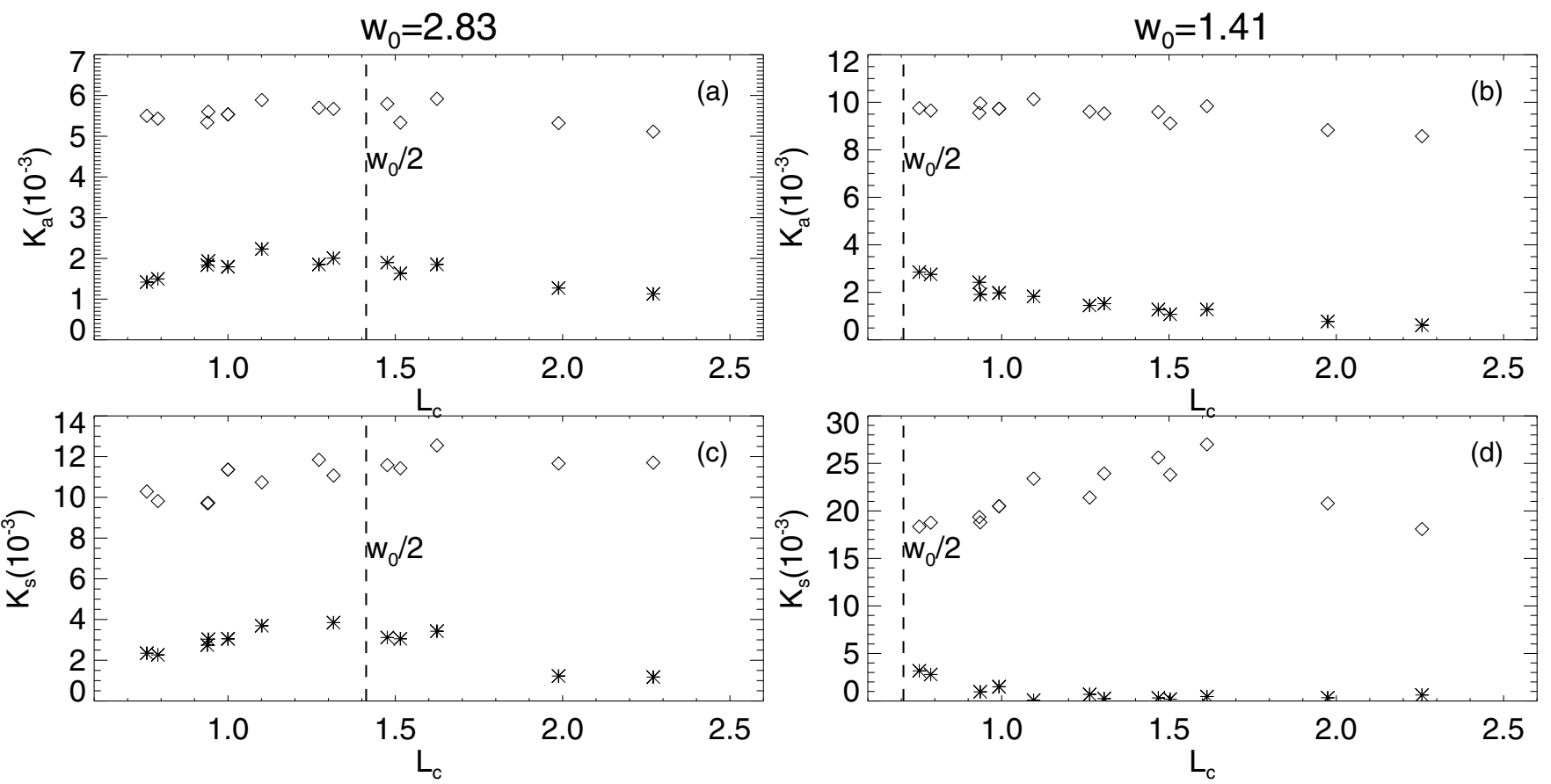

Figure 8. $K_{a}$ and $K_{s}$ as functions of the correlation length $L_{c}$ of the random structuring, with $\delta_{\rho}=0.18$ for the linear (asterisks) and nonlinear (diamonds) waves. Two sets of pulses were launched with $w_{0}=2.83(\mathrm{a}, \mathrm{c})$ or $w_{0}=1.41(\mathrm{~b}, \mathrm{~d})$.

attenuation and broadening occur for an initial pulse width $w_{0} \simeq 2.5$, which is approximately a value of $2 L_{c}$ (Figure 5(a) and (c)). To further verify this point, we launched a set of pulses in a random plasma with $L_{c}=2.0$ and expect maximum damping at $2 L_{c}=4.0$, which is out of the selected range of the initial pulse width. Indeed, Figure 5(b) and (d) exhibit no resonant attenuation. The turnover is only observed for linear pulses, suggesting that nonlinear steepening and broadening are stronger than the resonant attenuation effect. Narrower pulses are typically attenuated more than broader ones. We also find that the width broadening coefficient $K_{s}$ is negative in the vicinity of $w_{0} \simeq L_{c}$ for a linear pulse, meaning that the pulse width decreases as it propagates through the random plasma. This is due to the fact that a narrow pulse in this case tends to split into a main pulse and a co-evolving pulse, as shown in Figure 6(a) which shows two velocity profiles at $t=120$. This phenomenon may be considered to be the formation of an oscillatory wake behind the main pulse. This effect is also seen in the case of a broader pulse, while the main body of the pulse does not experience a major decrease in that case. A sufficiently broad pulse (Figure 6(b)) retains the structure of a single main pulse with multiple ripples on top of the main pulse. We performed a 
convergence test by switching to the PPM flux limiter. In both cases, the splitting of narrow pulses and the development of multiple ripples for broad pulses are clearly found. Thus, we can confidently conclude that these are physical effects caused by the randomly structured plasma alone.

\subsection{Effect of Random Structuring Density Contrast}

Next, we consider the dependence of $K_{a}$ and $K_{s}$ on the density contrast $\delta_{\rho}$. Figure 7(b) and (d) show that a larger density contrast typically causes stronger attenuation and dispersion for pulses with $w_{0}$ greater than $L_{c}$. However, in the case of narrow pulses (Figure 7(a) and (c)), a higher density contrast encourages the splitting of the pulse (see Figure 6) and thus gives zero or negative broadening. Therefore, a non-monotonic dependence occurs for $K_{s}\left(\delta_{\rho}\right)$ in the linear pulse region; small density contrasts are not strong enough to cause a pulse splitting result in positive broadening, and $K_{s}$ increases with $\delta_{\rho}$. If $\delta_{\rho}$ is large enough to cause pulse splitting, $K_{s}$ decreases with increasing $\delta_{\rho}$, even to zero or negative values (see Figure 7(c)). For the pulse amplitude, a higher $\delta_{\rho}$ usually results in a larger attenuation coefficient $K_{a}$. We note that $K_{a}$ and $K_{s}$ exhibit steeper gradients in the linear wave regime, suggesting that nonlinear waves are less sensitive to the random structuring.

\subsection{Effect of Random Structuring Correlation Length}

Finally, we examine the effect of the correlation length $L_{c}$ of the random structuring on the fast pulse behavior. Figure 8(a) and (c) show that $K_{a}$ and $K_{s}$ reach larger values for $L_{c} \simeq w_{0} / 2$ in the linear regime. This is in agreement with the results of Section 3.1. In the case of a pulse with a sufficiently narrow initial width, no clear resonance region is found (see Figure 8(b) and (d)).

\section{CONCLUSIONS}

We performed a parametric study on fast magnetoacoustic pulses propagating in a randomly structured plasma perpendicular to the magnetic field. The fast wave is initiated with the form of a single Gaussian pulse. During propagation, the main pulse excites secondary propagating and standing waves, thereby losing its energy. Due to the random structuring, the pulse experiences attenuation and broadening. Thus, a randomly structured plasma is effectively a dispersive medium in the context of pulse propagation. This effect is similar to the recently considered dispersive evolution of coronal fast wave trains (Yuan et al. 2013; Pascoe et al. 2013, 2014; Oliver et al. 2014; Nistico et al. 2014). In addition, even if the plasma is considered as being ideal, random structuring introduces effective dissipation of the wave that needs to be taken into account.

The most efficient interaction of linear low-amplitude pulses with the random structuring occurs when the wavelength is about twice the correlation length of the structuring. Nonlinear effects cause pulse steepening, which can modify this behavior. Observations of this effect could be used to estimate the correlation length of the structuring in the corona. In general, fine structuring causes effective attenuation and dispersion for propagating pulses, and acts against nonlinear steepening.

The evolution of the amplitude and width of the fast magnetoacoustic pulse was found to be well-approximated by simple linear functions of the travel distance, with the exception of the nonlinear large pulse amplitude profile, which was modeled as an exponential function. Several factors were identified to influence the amplitude attenuation coefficient $K_{a}$ and the width broadening coefficient $K_{s}$. A higher density contrast leads to faster attenuation and broadening of wider pulses. For narrower pulses, the splitting of the original pulse competes with dispersive broadening and thus the broadening coefficient may be zero or negative.

One interesting application of our results is connected with EIT wave studies. In the context of EIT wave evolution, the ideal plasma of the corona could be considered as an effectively dissipative and dispersive medium. Many EIT wave features are reproduced by our simulations. The pulse profile deviates from its initial Gaussian shape (see Figure 11 in Muhr et al. 2011), and generates an oscillatory wave, splits, or develops ripple structure at later stages (Liu et al. 2010; Shen \& Liu 2012). The effect of nonlinear steepening is also demonstrated in our simulations (Veronig et al. 2010). Scattering of the main pulse by non-uniformities leads to the excitation of secondary waves (Li et al. 2012; Shen \& Liu 2012; Olmedo et al. 2012; Shen et al. 2013; Yang et al. 2013) and propagation dips (Muhr et al. 2011). Our study is particularly relevant to interpreting the apparent decay of EIT waves (e.g., Ballai 2007; Long et al. 2011b), which could be caused by the combination of geometrical effects (e.g., wave propagation and expansion on spherical or cylindrical surfaces), non-ideal MHD effects (viscosity, resistivity, radiation, and thermal conduction) and scattering and dispersive broadening due to the random fine structuring discussed in this study. The amplitude and width evolution has been measured by a number of authors, e.g., Long et al. (2011b) and Shen \& Liu (2012). Although an apparently exponential decay in the wave amplitude was detected, only a simple linear fit has been attempted (Long et al. 2011b). Our simulations suggest that an exponential fit is more appropriate in nonlinear pulses. The profile broadening of EIT waves could be approximated by a linear function (Long et al. 2011a); however, zero or negative broadening occurs in some narrow pulses of finite amplitude (Warmuth et al. 2004b). Likewise, the analysis of nonlinear effects in EIT wave propagation (e.g., Afanasyev \& Uralov 2011) needs to account for the effective dispersion of the corona. Our modeling shows that a more detailed analysis of the amplitude and width evolution of EIT waves observed with high spatial and time resolution may allow one to fully exploit the plasma diagnostics potential.

Our model could be extended to two-dimensional and threedimensional MHD models. Multi-dimensional MHD simulations could investigate other physics in inhomogeneous plasmas and will allow mode conversion (Sedláček 1972; Ionson 1978; Hollweg \& Yang 1988; Ruderman \& Roberts 2002; Goossens et al. 2002). Also, line-tying boundary conditions will create fast magnetoacoustic resonators and a possibility for the excitation of standing modes (e.g., of the kink and sausage symmetry) in addition to the secondary propagating waves. Waves propagating through structures with low density contrasts, such as those we consider here for a finely structured corona, may demonstrate non-exponential damping behavior (Pascoe et al. 2012, 2013; Hood et al. 2013). Large local Alfvén speed gradients also allow phase mixing (Heyvaerts \& Priest 1983; Similon \& Sudan 1989; Ofman \& Aschwanden 2002), which may play an important role in dissipating a fast pulse in a finely structured plasma. Other non-ideal processes may also be enhanced by the large local gradients provided by fine structuring.

This work is supported by the National Basic Research Program of China via grant 2012CB825601 (D.Y. and B.L.), FWO Odysseus Programme (D.Y.), the Marie Curie PIRSES-GA2011-295272 RadioSun project, the European Research Council 
under the SeismoSun Research Project No. 321141 (D.Y., D.J.P., V.M.N.), the Open Research Program KLSA201312 of Key Laboratory of Solar Activity of National Astronomical Observatories of China (D.Y.), STFC consolidated grant ST/L000733/1, the BK21 plus program through the National Research Foundation funded by the Ministry of Education of Korea (V.M.N.), the National Natural Science Foundation of China (40904047, 41174154, and 41274176), and the Provincial Natural Science Foundation of Shandong via grant JQ201212 (B.L., D.Y.).

\section{REFERENCES}

Afanasyev, A. N., \& Uralov, A. M. 2011, SoPh, 273, 479

Arber, T. D., Longbottom, A. W., Gerrard, C. L., \& Milne, A. M. 2001, JCoPh, 171,151

Arregui, I., Andries, J., Van Doorsselaere, T., Goossens, M., \& Poedts, S. 2007, A\&A, 463, 333

Aschwanden, M. J., Nightingale, R. W., Andries, J., Goossens, M., \& Van Doorsselaere, T. 2003, ApJ, 598, 1375

Ballai, I. 2007, SoPh, 246, 177

Beresnyak, A., \& Lazarian, A. 2008, ApJ, 678, 961

Bogdan, T. J., \& Zweibel, E. G. 1985, ApJ, 298, 867

Bogdan, T. J., \& Zweibel, E. G. 1987, ApJ, 312, 444

Botha, G. J. J., Arber, T. D., Nakariakov, V. M., \& Zhugzhda, Y. D. 2011, ApJ, 728,84

Chen, P. F., Fang, C., \& Shibata, K. 2005, ApJ, 622, 1202

Chen, Y., Feng, S. W., Li, B., et al. 2011, ApJ, 728, 147

Chen, Y., Song, H. Q., Li, B., et al. 2010, ApJ, 714, 644

De Moortel, I., \& Nakariakov, V. M. 2012, RSPTA, 370, 3193

De Moortel, I., \& Pascoe, D. J. 2012, ApJ, 746, 31

Delannée, C. 2000, ApJ, 545, 512

Díaz, A. J., Oliver, R., \& Ballester, J. L. 2005, A\&A, 440, 1167

Edwin, P. M., \& Roberts, B. 1983, SoPh, 88, 179

Gallagher, P. T., \& Long, D. M. 2011, SSRv, 158, 365

Goedbloed, J. P., Keppens, R., \& Poedts, S. 2010, Advanced Magnetohydrodynamics: With Applications to Laboratory and Astrophysical Plasmas (Cambridge: Cambridge Univ. Press)

Goedbloed, J. P., \& Poedts, S. 2004, Principles of Magnetohydrodynamics: With Applications to Laboratory and Astrophysical Plasmas (Cambridge: Cambridge Univ. Press)

Goossens, M., Andries, J., \& Aschwanden, M. J. 2002, A\&A, 394, L39

Gruszecki, M., Murawski, K., Selwa, M., \& Ofman, L. 2006, A\&A, 460, 887

Harten, A., Lax, P. D., \& Leer, B. v. 1983, SIAMR, 25, 35

Heyvaerts, J., \& Priest, E. R. 1983, A\&A, 117, 220

Hollweg, J. V., \& Yang, G. 1988, JGR, 93, 5423

Hood, A. W., Ruderman, M., Pascoe, D. J., et al. 2013, A\&A, 551, A39

Ionson, J. A. 1978, ApJ, 226, 650

Keppens, R., Bogdan, T. J., \& Goossens, M. 1994, ApJ, 436, 372

Keppens, R., Meliani, Z., van Marle, A. J., et al. 2012, JCoPh, 231, 718

Keppens, R., \& Porth, O. 2014, JCoAM, 266, 87

Kleva, R. G., \& Drake, J. F. 1992, ApJ, 395, 697

Klimchuk, J. A. 2006, SoPh, 234, 41

Koren, B. 1993, in A Robust Upwind Discretization Method for Advection, Diffusion and Source Terms, ed. C. B. Vreugdenhil (Braunschweig: Vieweg), 117

Kuzmin, D. 2006, JCoPh, 219, 513

Labonte, B. J., \& Ryutova, M. 1993, ApJ, 419, 388

Lazzaro, E., Lontano, M., \& Ryutov, D. D. 2000, PhRvE, 61, 3069

Li, B., \& Li, X. 2007, ApJ, 661, 1222

Li, B., \& Li, X. 2008, ApJ, 682, 667

Li, H.-S., \& Zweibel, E. G. 1987, ApJ, 322, 248

Li, T., Zhang, J., Yang, S., \& Liu, W. 2012, ApJ, 746, 13

Liu, W., Nitta, N. V., Schrijver, C. J., Title, A. M., \& Tarbell, T. D. 2010, ApJL, 723, L53

Long, D. M., DeLuca, E. E., \& Gallagher, P. T. 2011a, ApJL, 741, L21

Long, D. M., Gallagher, P. T., McAteer, R. T. J., \& Bloomfield, D. S. 2011b, A\&A, 531, A42
Malara, F., De Franceschis, M. F., \& Veltri, P. 2003, A\&A, 412, 529

Markwardt, C. B. 2009, in ASP Conf. Ser. 411, Astronomical Data Analysis Software and Systems XVIII, ed. D. A. Bohlender, D. Durand, \& P. Dowler (San Francisco, CA: ASP), 251

Muhr, N., Veronig, A. M., Kienreich, I. W., Temmer, M., \& Vršnak, B. 2011, ApJ, 739, 89

Murawski, K., Nakariakov, V. M., \& Pelinovsky, E. N. 2001, A\&A, 366, 306

Nakariakov, V. M., \& Ofman, L. 2001, A\&A, 372, L53

Nakariakov, V. M., Pascoe, D. J., \& Arber, T. D. 2005, SSRv, 121, 115

Nakariakov, V. M., \& Verwichte, E. 2005, LRSP, 2, 3

Nistico, G., Pascoe, D., \& Nakariakov, V. 2014, A\&A, 569, A12

Ofman, L. 2005, SSRv, 120, 67

Ofman, L. 2010, LRSP, 7, 4

Ofman, L., \& Aschwanden, M. J. 2002, ApJL, 576, L153

Ofman, L., \& Thompson, B. J. 2002, ApJ, 574, 440

Ofman, L., \& Wang, T. 2002, ApJL, 580, L85

Oliver, R., Ruderman, M. S., \& Terradas, J. 2014, ApJ, 789, 48

Olmedo, O., Vourlidas, A., Zhang, J., \& Cheng, X. 2012, ApJ, 756, 143

Parnell, C. E., \& De Moortel, I. 2012, RSPTA, 370, 3217

Pascoe, D. J. 2014, RAA, 14, 805

Pascoe, D. J., Hood, A. W., de Moortel, I., \& Wright, A. N. 2012, A\&A, 539, A37

Pascoe, D. J., Nakariakov, V. M., \& Arber, T. D. 2007, SoPh, 246, 165

Pascoe, D. J., Nakariakov, V. M., \& Kupriyanova, E. G. 2013, A\&A, 560, A97

Pascoe, D. J., Nakariakov, V. M., \& Kupriyanova, E. G. 2014, A\&A, 568, A20

Pascoe, D. J., Wright, A. N., \& De Moortel, I. 2010, ApJ, 711, 990

Pascoe, D. J., Wright, A. N., \& De Moortel, I. 2011, ApJ, 731, 73

Patsourakos, S., \& Vourlidas, A. 2012, SoPh, 281, 187

Porth, O., Xia, C., Hendrix, T., Moschou, S. P., \& Keppens, R. 2014, ApJS, 214,4

Ruderman, M. S., \& Roberts, B. 2002, ApJ, 577, 475

Ryutova, M. P., \& Priest, E. R. 1993a, ApJ, 419, 349

Ryutova, M. P., \& Priest, E. R. 1993b, ApJ, 419, 371

Sakurai, T., Goossens, M., \& Hollweg, J. V. 1991, SoPh, 133, 227

Sedláček, Z. 1972, CzJPh, 22, 439

Shen, Y., \& Liu, Y. 2012, ApJ, 754, 7

Shen, Y., Liu, Y., Su, J., et al. 2013, ApJL, 773, L33

Similon, P. L., \& Sudan, R. N. 1989, ApJ, 336, 442

Stepanov, A. V., Zaitsev, V. V., \& Nakariakov, V. M. 2012, PhU, 55, 929

Suzuki, T. K., \& Inutsuka, S.-I. 2006, JGRA, 111, 6101

Thompson, B. J., Gurman, J. B., Neupert, W. M., et al. 1999, ApJL, 517, L151

Tóth, G., \& Odstrčil, D. 1996, JCoPh, 128, 82

Tsiklauri, D. 2014, PhPl, 21, 052902

Tsiklauri, D., Aschwanden, M. J., Nakariakov, V. M., \& Arber, T. D. 2004, A\&A, 419,1149

Valley, G. C. 1971, ApJ, 168, 251

Valley, G. C. 1974, ApJ, 188, 181

Van Doorsselaere, T., Brady, C. S., Verwichte, E., \& Nakariakov, V. M. 2008a, A\&A, 491, L9

Van Doorsselaere, T., Nakariakov, V. M., \& Verwichte, E. 2008b, ApJL, 676, L73

Van Doorsselaere, T., Wardle, N., Del Zanna, G., et al. 2011, ApJL, 727, L32

Veronig, A. M., Muhr, N., Kienreich, I. W., Temmer, M., \& Vršnak, B. 2010, ApJL, 716, L57

Wang, Y.-M. 2000, ApJL, 543, L89

Warmuth, A. 2011, PPCF, 53, 124023

Warmuth, A., Vršnak, B., Magdalenić, J., Hanslmeier, A., \& Otruba, W. 2004a, A\&A, 418, 1101

Warmuth, A., Vršnak, B., Magdalenić, J., Hanslmeier, A., \& Otruba, W. 2004b, A\&A, 418, 1117

Wu, S. T., Li, B., Wang, S., \& Zheng, H. 2005, JGRA, 110, 11102

Wu, S. T., Zheng, H., Wang, S., et al. 2001, JGR, 106, 25089

Yang, L., Zhang, J., Liu, W., Li, T., \& Shen, Y. 2013, ApJ, 775, 39

Yuan, D., \& Nakariakov, V. M. 2012, A\&A, 543, A9

Yuan, D., Nakariakov, V. M., Huang, Z., et al. 2014a, ApJ, 792, 41

Yuan, D., Shen, Y., Liu, Y., et al. 2013, A\&A, 554, A144

Yuan, D., Sych, R., Reznikova, V. E., \& Nakariakov, V. M. 2014b, A\&A, 561, A19 